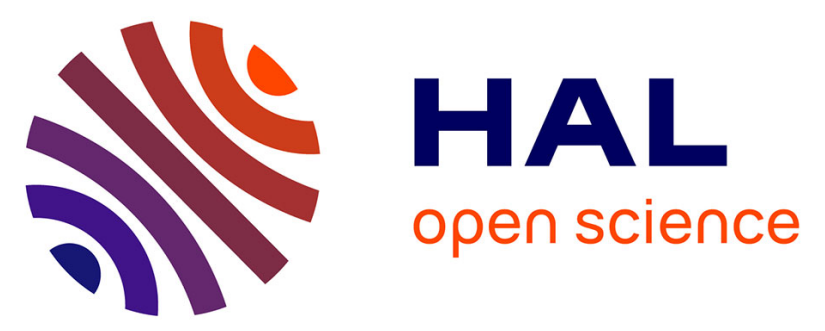

\title{
Unsteady coupling of Navier-Stokes and radiative heat transfer solvers applied to an anisothermal multicomponent turbulent channel flow
}

\author{
J. Amaya, O. Cabrit, Damien Poitou, B. Cuenot, Mouna El-Hafi
}

\section{- To cite this version:}

J. Amaya, O. Cabrit, Damien Poitou, B. Cuenot, Mouna El-Hafi. Unsteady coupling of Navier-Stokes and radiative heat transfer solvers applied to an anisothermal multicomponent turbulent channel flow. Journal of Quantitative Spectroscopy and Radiative Transfer, 2010, 111 (2, SI), pp.295-301. 10.1016/j.jqsrt.2009.06.014 . hal-01712150

\author{
HAL Id: hal-01712150 \\ https://hal.science/hal-01712150
}

Submitted on 8 Nov 2019

HAL is a multi-disciplinary open access archive for the deposit and dissemination of scientific research documents, whether they are published or not. The documents may come from teaching and research institutions in France or abroad, or from public or private research centers.
L'archive ouverte pluridisciplinaire HAL, est destinée au dépôt et à la diffusion de documents scientifiques de niveau recherche, publiés ou non, émanant des établissements d'enseignement et de recherche français ou étrangers, des laboratoires publics ou privés. 


\title{
Unsteady coupling of Navier-Stokes and radiative heat transfer solvers applied to an anisothermal multicomponent turbulent channel flow
}

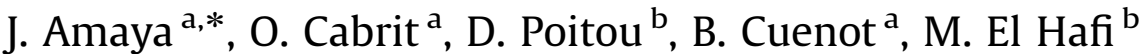 \\ ${ }^{\text {a } C E R F A C S}$ - 42, rue Gaspard Coriolis, 31057 Toulouse Cedex 01, France \\ ${ }^{\mathrm{b}}$ Centre RAPSODEE, Ecole des Mines d'Albi Carmaux, 81013 Albi Cedex 9, France
}

Keywords:

Discrete ordinates method

Direct numerical simulation

Turbulent channel

Non-gray gas radiation

\begin{abstract}
A B S T R A C T
Direct numerical simulations (DNS) of an anisothermal reacting turbulent channel flow with and without radiative source terms have been performed to study the influence of the radiative heat transfer on the optically non-homogeneous boundary layer structure. A methodology for the study of the emitting/absorbing turbulent boundary layer (TBL) is presented. Details on the coupling strategy and the parallelization techniques are exposed. An analysis of the first order statistics is then carried out. It is shown that, in the studied configuration, the global structure of the thermal boundary layer is not significantly modified by radiation. However, the radiative transfer mechanism is not negligible and contributes to the heat losses at the walls. The classical law-of-the-wall for temperature can thus be improved for RANS/LES simulations taking into account the radiative contribution.
\end{abstract}

\section{Introduction}

The thermal interaction between a fluid and a solid takes place at the interface, where energy is transported by three mechanisms: convection, conduction and radiation. While the first two mechanisms have been studied for nearly one century, only in the last decades models for the coupled interaction with radiation have been developed. These models have evolved, from the $1 \mathrm{D}$ gray gas approximation of Viskanta in 1963 [1] to the turbulence-radiation interaction channel simulations studied by Soufiani et al. in 1990 [2]. To the authors knowledge, this is the first time that a $3 \mathrm{D}$ unsteady coupling of direct numerical simulation (DNS) and gas radiation solvers, on a non-gray multicomponent gas, is performed. The present is an exploratory work and is intended to be a

\footnotetext{
* Corresponding author. Tel.: +335611930 08; fax: +33561193000.

E-mail addresses: amaya@cerfacs.fr, jarodz@hotmail.com (J. Amaya).
}

starting point in the research for a detailed comprehension of the fluid/wall thermal interactions.

In industrial applications for combustion systems, radiation is known to influence the behavior of the flow as shown by Schmitt et al. [3]. Conductive energy exchange occurs at a very small scale near the wall, which implies a fine resolution of the Navier-Stokes solver, and consequently a rise in the computational cost. One way to avoid these problems is to construct a model that predicts the behavior of the boundary layer, from quantities available in coarser meshes. DNS are often used to study and derive such models for LES and RANS applications, as it resolves all mechanisms to the smallest active scale.

\subsection{Case of study}

As presented in Fig. 1, the classic computational domain for turbulent minimal channel flow DNS is used [4,5] at a friction Reynolds number of $\operatorname{Re}_{\tau}=400$ which correspond to a Reynolds number of $R e=7530$. Periodic 


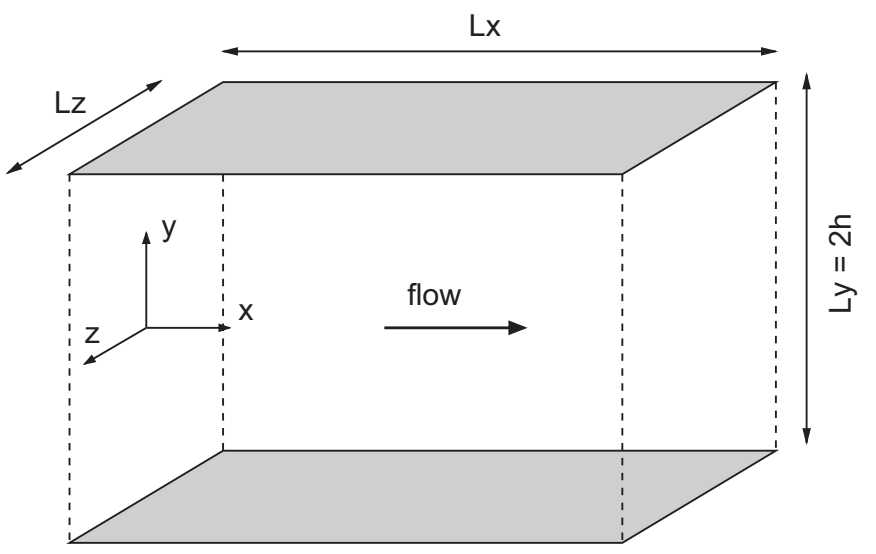

Fig. 1. Box dimensions of the computational domain: $L x=3.5 h, L y=2 h, L z=1.3 h$.

boundary conditions are applied, in the Navier-Stokes solver, in the streamwise $(\vec{x})$ and spanwise $(\vec{z})$ directions. Moreover, the streamwise flow is enforced by adding a space/time constant source term to the momentum conservation equation, while a volume source term that warms the fluid is added to the energy conservation equation to sustain the bulk temperature, $T_{b}=2000 \mathrm{~K}$, as presented in [6]. Finally, a no-slip isothermal boundary condition is used at the wall with a constant temperature of $T_{w}=1750 \mathrm{~K}$.

The computational domain is composed of 292142 hexahedral elements constructed from 307824 grid points $(44 \times 159 \times 44$, in $x, y$ and $z)$. Grid spacing in viscous wall units is setup to be at least half the step size of the minimal channel flow DNS of Jiménez and Moin [4], namely $\Delta x^{+} \approx 32$ in the $x$ direction and $\Delta z^{+} \approx 12$ in the $z$ direction. In the wall-normal direction grid spacing is refined in the near wall zone: the first point off the wall is at $y^{+} \approx 0.9$ and the maximum spacing is $\Delta y_{\max }^{+} \approx 6.5$.

The gas used in the present work is a mixture of seven reacting species typical of many industrial applications in the energy sector: $\mathrm{CO}, \mathrm{CO}_{2}, \mathrm{H}, \mathrm{H}_{2}, \mathrm{H}_{2} \mathrm{O}, \mathrm{OH}$ and $\mathrm{N}_{2}$.

\subsection{Coupling flow equations with radiation}

Radiation and flow dynamics are coupled through the radiative heat flux term $q_{i}^{\text {rad }}$ in the multispecies reacting total non-chemical energy conservation equation

$\frac{\partial \rho E}{\partial t}+\frac{\partial}{\partial x_{i}}\left(\rho u_{i} E\right)=\dot{\omega}_{T}-\frac{\partial}{\partial x_{i}}\left(q_{i}^{\text {gas }}+q_{i}^{r a d}\right)$

$+\frac{\partial}{\partial x_{j}}\left(\sigma_{i j} u_{i}\right)+u_{i} \mathscr{S}_{m}+\mathscr{Q}$

In this expression $i$-subscripted quantities refer to variables dependent on directions, $\rho$ is the density of the gas, $E$ is the total energy, $u$ is the velocity, $\dot{\omega}_{T}$ is the heat release due to the chemical reaction, $q_{i}^{\text {gas }}$ is the heat flux of the gas, $q_{i}^{\text {rad }}$ is the radiative heat flux, $\mathscr{S}_{m}$ is the momentum source term, $\mathscr{Q}$ is the energy source term and $\sigma_{i j}$ is the total stress tensor. The divergence of the radiative heat flux is called the radiative source term $\mathscr{S}_{r}$, and can be obtained using the conservation equation of radiative energy [7]:

$\frac{\partial}{\partial x_{i}}\left(q_{i}^{r a d}\right)(\mathbf{x})=\mathscr{S}_{r}(\mathbf{x})=\int_{0}^{\infty} \kappa_{v}\left(4 \pi I_{b v}(\mathbf{x})-G_{v}(\mathbf{x})\right) d v$

$G_{v}(\mathbf{x})=\int_{4 \pi} I_{v}\left(\mathbf{x}, \mathbf{s}_{\mathbf{i}}\right) d \Omega_{i}$

where $\mathbf{x}$ is a location vector, $\kappa$ is the absorption coefficient, $v$ is the frequency, $I_{b v}$ is the blackbody emitted intensity and $G_{v}$ is the sum of the incident intensities coming from all solid angle directions $\mathbf{s}_{\mathbf{i}}$, expressed by relation (3). Eq. (2) states that the radiative source term in an infinitesimal volume around $\mathbf{x}$, is equal to the difference between the emitted and the absorbed incident radiation.

\section{Numerical tools}

\subsection{Navier-Stokes solver}

DNS have been computed using AVBP [8]. This LES/DNS code has been designed to solve the full 3D compressible reacting Navier-Stokes equations on structured, unstructured and hybrid meshes. Numerous publications have shown the efficiency of this code. ${ }^{1}$ In the present work, a third order in time and fourth order in space Galerkin finite element numerical scheme has been used.

\subsection{Radiative heat transfer solver}

Eq. (2) is calculated using DOMASIUM [9], a code that resolves the $3 \mathrm{D}$ radiative heat transfer equation over structured, unstructured and hybrid meshes, using the discrete ordinates method [7]. The main features of the code include:

- Intensities at the faces of each unstructured cell can be computed using exponential $[10,11]$ or diamond mean flux schemes [12].

- An angular discretization is used to integrate the incident intensity $G_{v}$ over all the solid angles $\Omega$. Possible angular quadratures includes $S_{N}, T_{N}$ and $L C_{11}$ [13].

\footnotetext{
${ }^{1}$ http://www.cerfacs.fr/cfd/CFDPublications.html.
} 
- Spectral integration can be performed using narrow band (SNB-CK) [14] or global spectral models (FSSNBcK [15], WSGG[16]).

- Boundary conditions are characterized by the emissivity $\varepsilon_{w}$, which can vary from $\varepsilon_{w}=1$ (black walls) to $\varepsilon_{w}=$ 0 (reflective walls). When using the narrow band model, the boundary emissivity can also be setup to vary with the frequency: $\varepsilon_{w}=\varepsilon_{w}(v)$.

Parallelization is done by direction/frequency or direction/domain decomposition, depending on the spectral model used. This allows to solve complex geometries on massively parallel computers, and reduces the overall computational cost.

\subsubsection{Direction/frequency parallelization}

This method is essentially used for the narrow band model, SNB-cK. The terms of the integral in (2) are decorrelated (in space and frequency), so each processor can independently calculate the intensities of a cell and add their contribution to the neighbor cells. Integration is done by addition of all the calculated intensities at the end of the cycle. Memory and CPU resources are best used when each processor computes a minimum number of directions.

As shown in Fig. 2 (left), when the number of processors $P_{r}=10$ is superior to the number of directions $N_{d i r}=5$, each processor calculates a fraction of the total narrow bands in one direction. In this particular example, each processor computes $B_{d}=N_{d i r} / P_{r}=0.5$ directions. The solver can handle any positive real value of $B_{d}$. Tests have shown a super linear speedup, most probably due to the cache effect.

\subsubsection{Direction/domain parallelization}

When a spectral model uses band groupement, as in the FS-SNBcK and WSGG methods, every processor needs to access the full spectrum data. The problem is then divided in two tasks: (a) calculate the emitted intensities of each cell in the domain and (b) add the contributions of each cell to the neighbor in a given direction for angular integration.
Fig. 2(right) shows how task (a) can be performed by domain decomposition (emitted intensity only requires the cell temperature, the pressure and the species mass fractions), and task (b) is done independently by each processor.

Here $B_{d}$ can only take positive integer values, so if the number of processors is superior to the number of directions, some processors may stay idle during the angular integration. Tests have shown that task (a) takes much more computational time than task (b), but increasing the number of processors will eventually lead to a stagnation of the speedup factor. Idle processors remain an issue. New parallelization techniques are currently being studied to avoid this problem.

\subsection{Coupling strategy}

During the coupled simulation the Navier-Stokes solver needs to know the radiative source term $\mathscr{S}_{r}$, and the radiation solver needs to know the temperature $T$, the pressure $P$ and the species molar concentration fields $X_{k}$.

Fig. 3(a) shows how the data can be exchanged, in a mode called sequential coupling strategy (SCS), where all the computing resources are allowed alternatively between the two codes. Another solution is to use a parallel coupling strategy (PCS) where both solvers run together sharing computational resources and using the data obtained from the last coupling iteration as shown in Fig. 3(b) [17]. In the present work the PCS was used.

A proper coupling strategy should acknowledge a good synchronization in physical an CPU time.

\subsubsection{Synchronization in physical time}

In compressible flow dynamics acoustics limits the simulation time step to the Courant-Friedrichs-Lewy (CFL) condition: $\Delta t_{C F L}=C F L \cdot \Delta x /(|\bar{u}|+c)$, while the radiative source term changes only about every convective characteristic time $\Delta t_{c}=\Delta x /|\bar{u}|$, where $\Delta x$ is the mesh step, $c$ is the sound speed and $\bar{u}$ the mean velocity. This time difference allows to simulate $N_{i}=\Delta t_{c} / \Delta t_{C F L}$ flow steps in the DNS code for each iteration of the radiation solver [18].
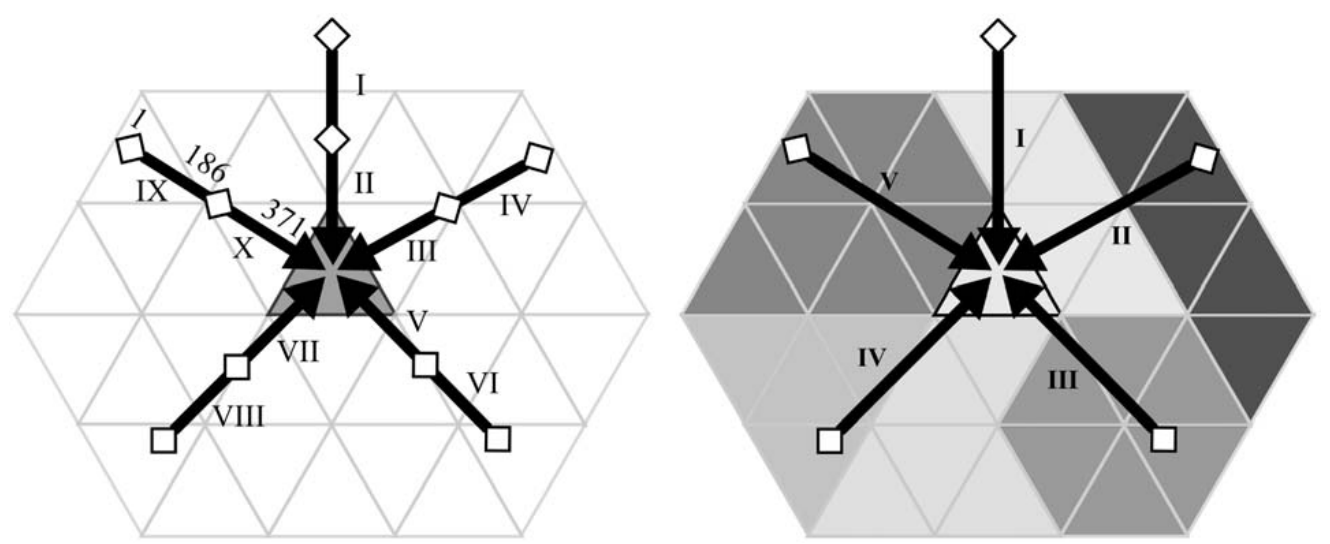

Fig. 2. Left: direction/frequency parallelization. In this example for each cell of the domain, 10 processors, noted from I to X, calculate five directions. Each processor computes only half of the spectrum in one direction. Right: direction/domain parallelization. In this case with six processors, emitted intensities are first calculated by domain decomposition using all available processors, then the five directions are integrated using only five processors (one processor must stay idle during angular integration). 
a

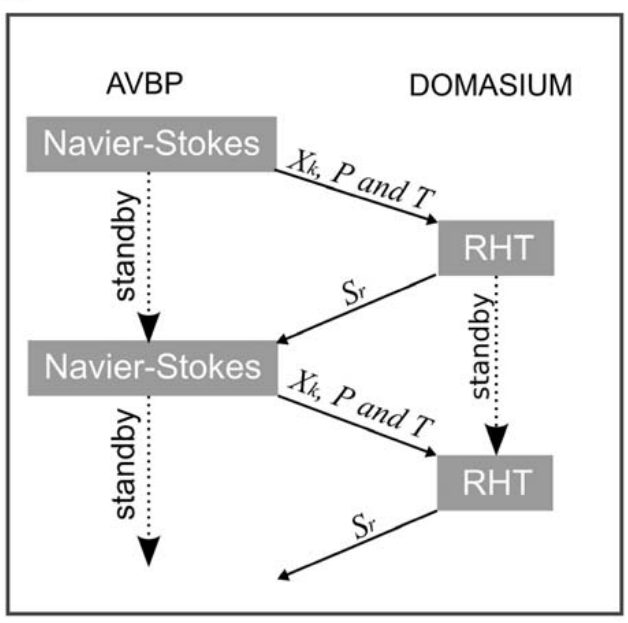

b

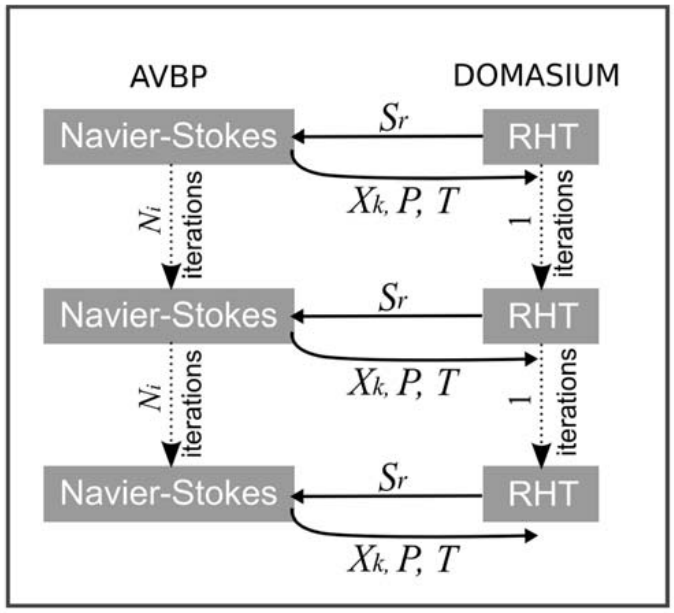

Fig. 3. (a) Sequential coupling strategy (SCS) and (b) parallel coupling strategy (PCS).

\subsubsection{Synchronization in CPU time}

The time between two coupling iterations must guarantee that $N_{i}$ flow iterations are computed for each radiation iteration: $N_{i} \cdot T_{f}=T_{r}$, where $T_{f}$ and $T_{r}$ are the overall computational time of 1 iteration of each code. These values depend on the number of processors allowed:

$T_{f}=\alpha_{f}\left(P_{f}\right) T_{f}^{1} / P_{f}$

$T_{r}=\alpha_{r}\left(P_{r}\right) T_{r}^{1} / P_{r}$

where $T_{*}^{1}$ is the computational time on 1 processor, $P_{*}$ is the number of processors allowed and $\alpha_{*}\left(P_{*}\right)$ is the speedup factor. In a perfectly scaling code, this last value can be considered equal to 1 . Combining these expressions and knowing that the total number of processors allowed is $P=P_{f}+P_{r}$, a balanced distribution of the processors can be obtained as

$P_{f}=\frac{P \cdot T_{f}^{1}}{T_{r}^{1} / N_{i}+T_{f}^{1}}$

Because of the fine resolution of the DNS, in the present work each radiative iteration was calculated every nine flow iterations. Communications between the DNS and the radiation solver are managed by a dynamic coupling software, called PALM [19].

\section{Selection of the radiation model}

On unsteady coupled simulations, the radiation methods must respond to two constrains: (a) the results must approach the reference data and (b) the computational cost must be low. While steady coupled simulations can be performed using only (a), unsteady applications, as for instance DNS or LES of combustion systems, need both (a) and (b).

Three aspects were studied when selecting the radiative models: the optical thickness of a 1D gas mixture layer of size $h$, the spectral behavior of the different models, and the angular discretization of the full domain.

\subsection{Optical thickness}

The mixture's absorption coefficient and the characteristic length of the configuration are used to determine the interaction distance of the radiated energy and the gas. The expression $A(h)=1-e^{-\bar{\kappa} h}$ determines the fraction of energy absorbed by a gas with a mean absorption coefficient $\bar{\kappa}$, at a distance $h$. The exponent $\tau=\bar{\kappa} h$ is the optical thickness of the gas. In a first approximation, the mean Planck absorption coefficient $\overline{\kappa_{P}}$, described by equation (7) [7], is used to calculate the optical thickness and the absorption properties of the mixture.

$\overline{\kappa_{P}}=\frac{\pi}{\sigma T^{4}} \int_{0}^{\infty} \kappa_{v} I_{b v} d v$

Eq. (7) is solved by numerical integration using the SNB approximation [14]. A value for the optical thickness of the channel was calculated: $\tau_{h} \approx 0.0066 \ll 1$. This value indicates that less than $1 \%$ of the energy emitted at the center of the channel will be absorbed by the gas. The optical thickness of the channel indicates that most of the radiated energy will impact the walls before getting absorbed by the medium. An increment of the wall heat flux should be detected, but no major modifications of the temperature fields are expected.

\subsection{The spectral models}

Using the narrow band approximation, at the distance $h$, a non-scattering gas column that only emits and absorbs in one optical path will produce in a narrow band a mean intensity that can be expressed using the following relations:

$$
\begin{aligned}
\langle I(h)\rangle_{\Delta v} & =\int_{\Delta v} T_{v}(h) I_{b v} d v=\left\langle I_{b}\right\rangle_{\Delta v} \int_{\Delta v} T_{v}(h) d v \\
& =\left\langle I_{b}\right\rangle_{\Delta v} \Delta v\langle T(h)\rangle_{\Delta v}
\end{aligned}
$$

Quantities that are averaged over a narrow band are described using the operator $\langle\cdot\rangle_{\Delta v}$. An accurate value for the mean transmissivity of the narrow band $\langle T(h)\rangle_{\Delta v}$ can be calculated using the Malkmus model (10). This value is 
then used to solve Eq. (9). The numerical solver is also used to integrate Eq. (8) using expression (11).

$$
\begin{aligned}
& \langle T(h)\rangle_{\Delta v}=\exp \left[\Phi_{\Delta v}\left(1-\left(1+\frac{2 \overline{\kappa_{\Delta v}} h}{\Phi_{\Delta v}}\right)^{1 / 2}\right)\right] \\
& T_{v}(h)=e^{-\kappa_{v} h}
\end{aligned}
$$

Here, the envelope of the absorption coefficient $\kappa_{v}$ in the narrow band $\Delta v$ is reconstructed by using the SNB parameters $\overline{\kappa_{\Delta v}}$ and $\Phi_{\Delta v}$ [20]. Both parameters are obtained by experimental methods [21].

A solver that integrates Eqs. (8) and (9) over 371 narrow bands was developed. 1D computations were carried up to test the performance of the different models supported by the radiation solver. The Malkmus transmittance model was used as a reference. Fig. 4 shows that, between 1750 and $2000 \mathrm{~K}$, temperature range of the channel simulation, the best results are obtained with the SNB-cK method with a high spectral quadrature. However, this method is computationally expensive. The FS-SNBcK method showed a good agreement with the Malkmus model. FS-SNBcK computations present the advantage of saving memory and CPU time, which is desirable for unsteady coupling with the Navier-Stokes solver. This latter method one was the retained for the present calculations.

\subsection{Angular quadrature}

Selection of the angular discretization was done by comparing the radiative fields (source term, heat flux vector field and wall heat fluxes) obtained with three different angular quadratures on an instantaneous solution of the turbulent channel: $L C_{11}$ (96 directions), $S_{8}$ (80 directions) and $S_{4}$ (24 directions). No significant differences were detected, therefore, $S_{4}$ quadrature was retained. On the boundaries where periodic conditions are applied in the Navier-Stokes computation, fully reflective boundary conditions are imposed in the radiative code. Emissivity of the solid walls is set to $\varepsilon_{w}=1$. These ensures the statistical homogeneity of the radiation fields in the streamwise and spanwise directions, without restricting the 3D spatial integration. As expected, these method guarantees a null flux through every plane perpendicular to the homogeneous directions.

\section{Results}

In this section all profiles shown are obtained by a first order statistical treatment: the solutions are averaged in time, and on the resulting fields spatial averages are performed over the homogeneous directions $(\vec{x}$ and $\vec{z})$. For any variable $f$, the quantity $\bar{f}$ represents ensemble average; $\{f\}$ represents the Favre average defined for a quantity $f$ as $\{f\}=\overline{\rho f} / \bar{\rho}$, the single prime (') and the double prime (") represent the turbulent fluctuations with respect to Reynolds and Favre averages, respectively.

Fig. 5(left) shows the profiles of three simulations in standard wall units $T^{+}=\left(T_{w}-\bar{T}\right) / T_{\tau}$ with $T_{\tau}=q_{w} /$ $\left(\rho_{w} C_{p, w} u_{\tau}\right)$ : the first is a test simulation without chemical reactions and without radiation, the second is a multicomponent reactive simulation without radiation and the third is a coupled multicomponent simulation with radiation. In the first simulation the temperature behaves like a passive scalar and tends towards the Kader logarithmic law [22]. This simulation is used to confirm the validity of the numerical method. Profiles of the two other simulations differ from the first one: the second simulation because of the multicomponent terms arising inside the Navier-Stokes equations [23] (this simulation constitutes the reference case for the coupled computation), and the third one because of both the multicomponent terms and the radiative transfer. Fig. 5(left) shows how the coupled multicomponent simulation differs from the reference values.

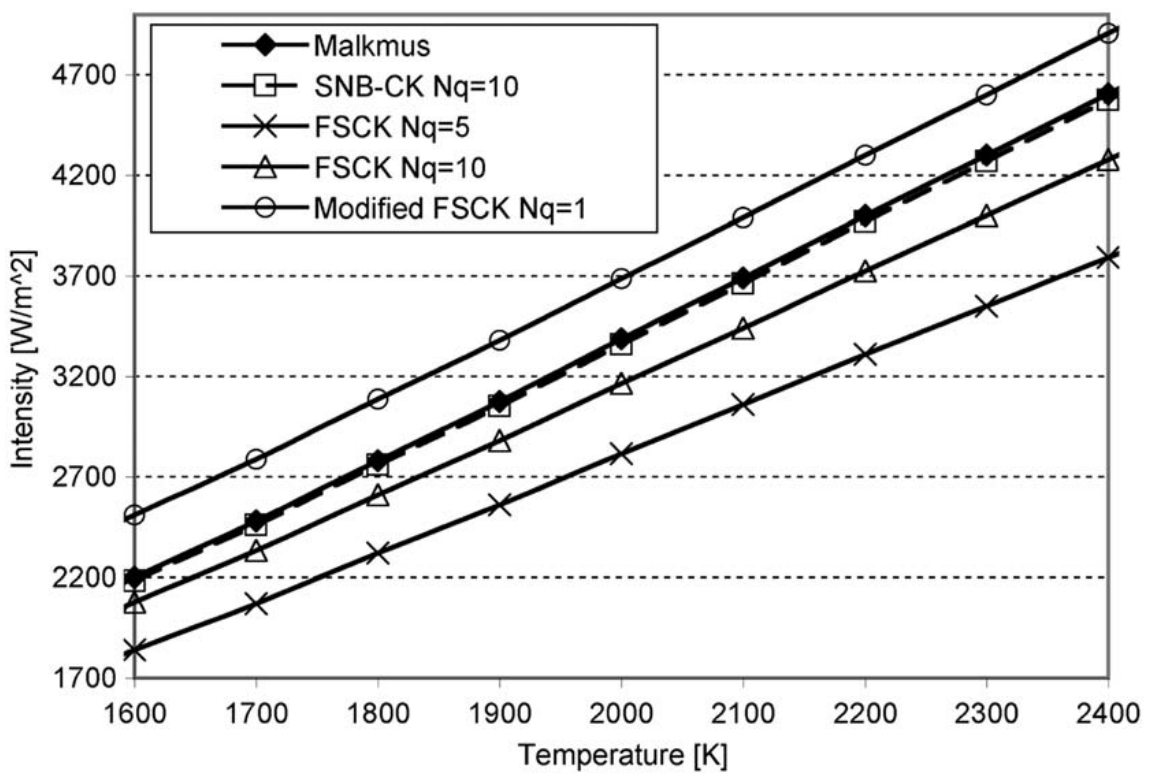

Fig. 4. Intensity at the exit of an isothermal gas column. Nine temperatures where tested using five spectral methods. 
a

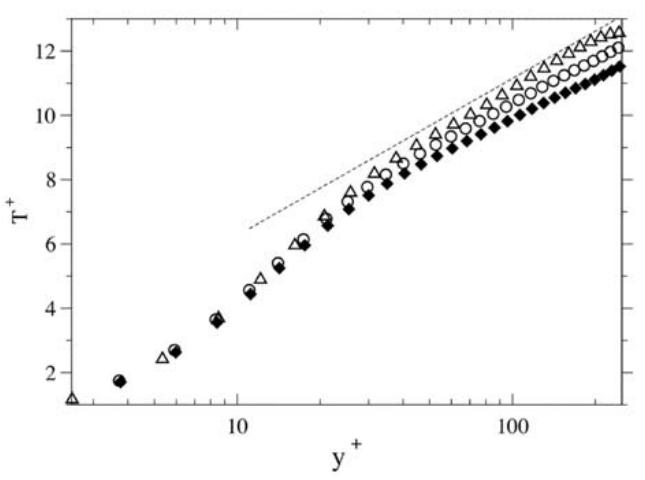

b

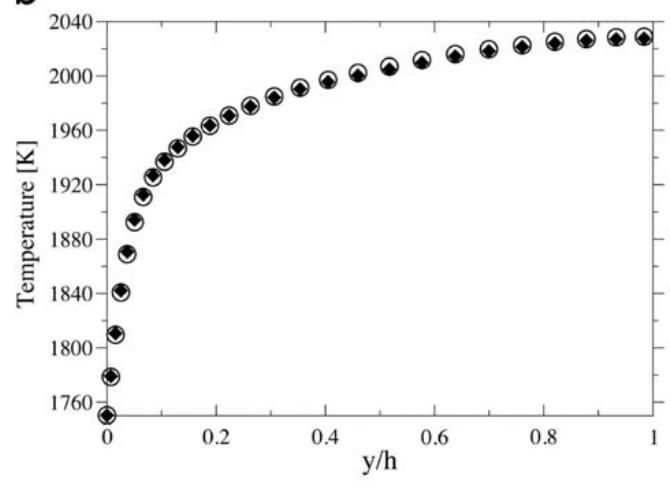

Fig. 5. Left: mean temperature in wall units. Right: mean temperature profile across the channel. Three simulations are represented by the following symbols: $\triangle$, non-reactive simulation without radiation; $\bigcirc$, multicomponent simulation without radiation; $\bullet$, coupled multicomponent simulation with radiation; - - - - Kader logarithmic law for $R e \rightarrow \infty$.

Fig. 5(right) shows that the mean temperature is similar for both the reference and the coupled simulations. This implies that the heat flux at the wall is the main quantity modified by the inclusion of radiation. A better understanding of these results is obtained by close inspection of the terms involved in the heat diffusion inside the boundary layer.

In a multicomponent reacting turbulent channel simulation, the energy conservation (1) can be expressed in terms of the specific enthalpy conservation ${ }^{2}$ :

$\rho \frac{D h}{D t}=\frac{D p}{D t}+\tau_{i j} \frac{\partial u_{i}}{\partial x_{j}}-\frac{\partial q_{i}}{\partial x_{i}}+\mathscr{Z}$

Applying statistical procedure to Eq.(12) leads to the following expression:

$\frac{\partial \bar{\rho}\left\{v^{\prime \prime} h^{\prime \prime}\right\}}{\partial y}=\overline{v \frac{\partial p}{\partial y}}+\overline{\tau_{i y} \frac{\partial u_{i}}{\partial y}}-\frac{\overline{\partial q_{y}}}{\partial y}+\overline{\mathscr{Q}}$

Neglecting the power of the pressure forces $\overline{v d p / d y}$ and the viscous effects $3 \overline{\tau_{i y} d u_{i} / d y}$, Eq. (13) can be rearranged as

$\frac{d q_{\text {tot }}}{d y} \approx \frac{d}{d y}(\underbrace{\bar{\rho}\left\{v^{\prime \prime} h_{s}^{\prime \prime}\right\}+\bar{\rho} \sum_{k}\left\{v^{\prime \prime} Y_{k}^{\prime \prime}\right\} \Delta h_{f, k}^{0}}_{\bar{q}_{y}^{\text {turb }}}+\underbrace{\bar{\rho}\left\{h_{k} Y_{k} V_{k, y}\right\}}_{\overline{q_{y}^{\text {multi }}}} \underbrace{\overline{-\lambda \frac{d T}{d y}}}_{\overline{q_{y}^{\text {Fourier }}}}-\overline{q_{y}^{\text {rad }}})$

where $q_{\text {tot }}$ is the total heat flux, $\overline{q_{y}^{\text {turb }}}$ is the turbulent flux of specific enthalpy, $\overline{q_{y}^{\text {multi }}}$ is the multicomponent laminar flux and $\overline{q_{y}^{\text {Fourier }}}$ is the Fourier flux. These three terms will be compared to the radiative flux term $\overline{q_{y}^{r a d}}$. The mean value of the energy source term $\overline{\mathscr{Q}}$ is constant in the wallnormal direction, implying that the total heat flux $q_{\text {tot }}(y)$ is represented by a line with a slope equal to $\overline{2}$.

Fig. 6 shows the four heat fluxes normalized by the Fourier flux at the wall $\left\|\overline{q_{w}^{\text {Fourier }}}\right\|$. It can be seen that the coupled and non-coupled heat fluxes remain almost the same, indicating the negligible coupling between the Navier-Stokes and the radiation equations.

\footnotetext{
${ }^{2}$ Ref. [24] gives details on the different forms of the energy transport equation.

${ }^{3}$ In the present simulation a low Mach number of $M=0.2$ was used.
}

In the reference simulation only the Fourier flux contributes to the total heat flux at the wall, whereas in the coupled simulation both the Fourier and the radiative fluxes contribute to it. The shape of the Fourier heat fluxes in both simulations is unchanged, and the relative error between their value at the wall is less than $1 \%$. This implies that changes in the slope of the total heat flux in the coupled simulation is mainly due to the radiative heat flux, which accounts for about 7\% of the total heat flux.

The profile of $\overline{q_{y}^{r a d}}$ is linear in this particular simulation, which indicates that the boundary data can be predicted from off-layer data in coarser meshes or directly from the radiation solver, whenever the optical thickness of the turbulent boundary layer (TBL) remains low.

In the context of LES or RANS simulations, this means that for optically thin TBL with low temperature gradients, usual law-of-the-wall may be applied, provided that the term $q_{w}^{r a d}$ is added to the total heat flux of the gas at the wall. The quantity $q_{w}^{\text {rad }}$ may be directly obtained using the radiation solver and can be injected without any special treatment on the law-of-the-wall of the Navier-Stokes solver.

\section{Conclusions and perspectives}

In the studied case, representing a low optical thickness and low temperature gradient configuration, no coupling was detected between the radiation term and the other energy conservation contributions.

Regarding the present database, it is not necessary to resolve the coupled Navier-Stokes/radiation equations in the turbulent boundary layer to capture the correct physics of the energy transfer to the wall. Indeed, this task could be very expensive in the context of RANS/LES applications for which law-of-the-wall treatment already gives reasonable results at convenient costs. The contribution of the radiative heat fluxes at the boundaries can be independently calculated using a radiation solver and added to the flux prediction of the law-of-the-wall used in the RANS/LES simulation. For instance in the present case, such a method could have improved the heat flux prediction of $7 \%$. 


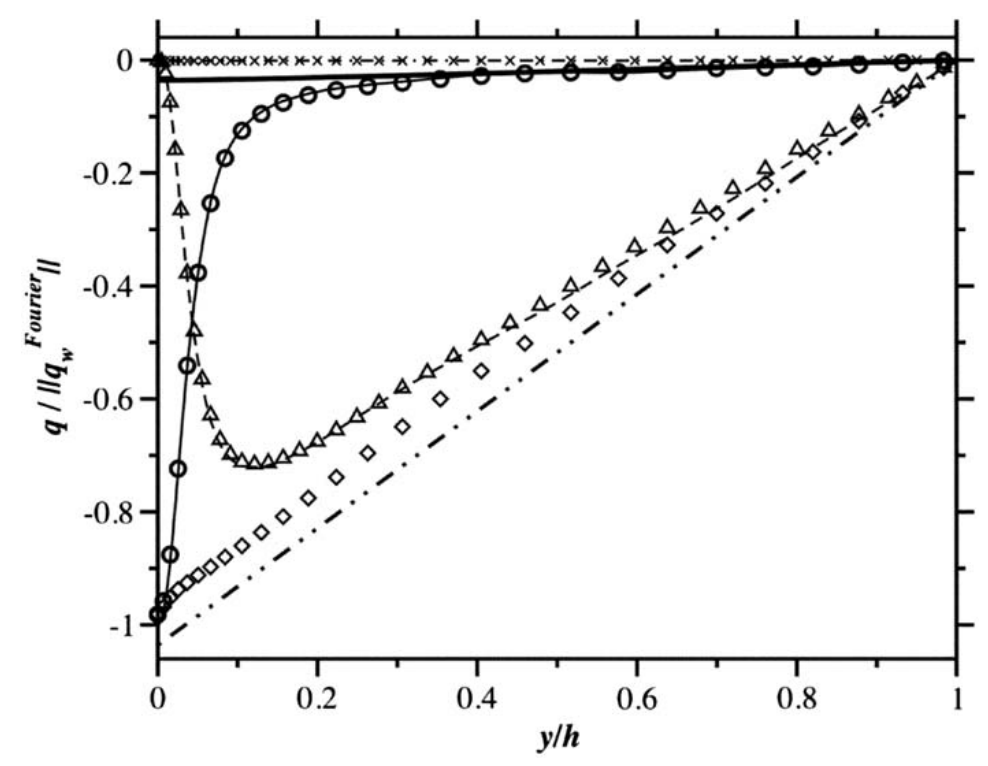

Fig. 6. Heat flux balance scaled by the modulus of the Fourier flux at the wall $\left\|\overline{q_{w}^{\text {Fourier }}}\right\|$. Symbols correspond to simulations without radiation, and lines to coupled multicomponent simulations with radiation. $-\cdots-$ and $\diamond$ : total heat flux, $q_{\text {tot }} ;-\frac{}{-}$ and $\bigcirc$ : Fourier heat flux, $\overline{q_{y}^{\text {Fourier }}}$; - . - and $\times$ :

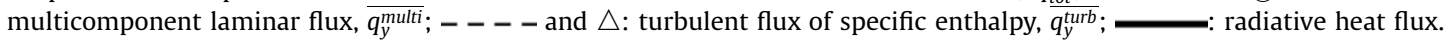

To generalize this conclusion to different kinds of mixture/temperature gradients boundary layers, simulations with strong temperature variations and higher optical thickness will be investigated.

\section{Acknowledgments}

The authors would like to acknowledge the National Research Agency of France (ANR) and the EM2C laboratory at Ecole Centrale Paris for their help.

\section{References}

[1] Viskanta R. Interaction of heat transfer by conduction, convection, and radiation in a radiating fluid. Journal of Heat Transfer 1963;85:318-28.

[2] Soufiani A, Mignon P, Taine J. Radiation-turbulence interaction in channel flows of infrared active gases. In: Proceedings of the ninth international heat transfer conference, vol. 6. Washington, DC: Hemisphere; 1990 . p. $403-8$

[3] Schmitt P, Poinsot TJ, Schuermans B, Geigle K. Large-eddy simulation and experimental study of heat transfer, nitric oxide emissions and combustion instability in a swirled turbulent high pressure burner. Journal of Fluid Mechanics 2007;570:17-46.

[4] Jiménez J, Moin P. The minimal flow unit in near-wall turbulence. Journal of Fluid Mechanics 1991;225:213-40.

[5] Kim J, Moin P, Moser R. Turbulence statistics in fully developed channel flow at low Reynolds number. Journal of Fluid Mechanics 1987;177:133-66.

[6] Cabrit O, Artal L, Nicoud F. Direct numerical simulation of turbulent multispecies channel flow with wall ablation. In: 39th AIAA thermophysics conference, AIAA Paper 2007-4401.

[7] Modest M. Radiative heat transfer. 2nd ed. San Diego: Academic Press; 2003.

[8] Schönfeld T, Rudgyard M. Steady and unsteady flows simulations using the hybrid flow solver AVBP. AIAA Journal 1999;37(11): 1378-85.

[9] Joseph D. Modelisation des transferts radiatifs en combustion par methode aux ordonees discretes sur des maillages non structures tridimensionels. Phd thesis, INP Toulouse; 2004.
[10] Sakami M, Charette A. A new differencing scheme for the discrete ordinates method in complex geometries. Revue degénérale de thermique 1998;37:440-9.

[11] Sakami M, Charette A, LeDez V. Radiative heat transfer in 3dimensional enclosures of complex geometry by using the discrete ordinates method. Journal of Quantitative Spectroscopy and Radiative Transfer 1998;56(4):517-33.

[12] Ströle J, Schnell U, Hein K. A mean flux discrete ordinates method interpolation scheme for general coordinates. In: 3rd international conference on heat transfer (Antalaya), 2001.

[13] Koch R, Becker R. Evaluation of the quadrature schemes for the discrete ordinates method. In: Elsevier (Ed.), Eurotherm73 on computational thermal radiation in participating media, vol. 11; 2003. p. 59-74.

[14] Liu F, Gulder O, Smallwood G, Ju Y. Non-gray gas radiative transfer analyses using the statistical narrow-band model. International Journal of Heat and Mass Transfer 1998;41:2227-36.

[15] Perez P, Joseph D, Haffi ME. Mise en place d'un modèle rapide de propriétés radiatives des gaz pour le couplage rayonnementcombustion. In: 13émes Journées Internationales de Thermique, 2007.

[16] Hottel HC, Sarofim AF. Radiative transfer. New York: McGraw-Hill; 1967.

[17] Duchaine F, Mendez S, Nicoud F, Corpron A, Moureau V, Poinsot T. Coupling heat transfer solvers and large eddy simulations. In: Proceedings of the summer program. Center for Turbulence Research: A Ames/Stanford University; 2008.

[18] dos Santos RG. Large eddy simulations of combustion including radiative heat transfer, Phd thesis, Ecole Centrale de Paris; 2007.

[19] Piacentini A. PALM: a dynamic parallel coupler. In: VECPAR, 2002, p. 479-92.

[20] Malkmus W. Random Lorentz band model with exponential-tailed s-1 line-intensity distribution function. Journal of the Optical Society of America 1967;57:323-9.

[21] Soufiani A, Taine J. High temperature gas radiative property parameters of statistical narrow-band model for $\mathrm{H}_{2} \mathrm{O}, \mathrm{CO}_{2}$ and $\mathrm{CO}$, and correlated-k model for $\mathrm{H}_{2} \mathrm{O}$ and $\mathrm{CO}_{2}$. International Journal of Heat and Mass Transfer 1997;40:987-91 [Technical note].

[22] Kader BA. Temperature and concentration profiles in fully turbulent boundary layers. International Journal of Heat and Mass Transfer 1981;24(9):1541-4.

[23] Giovangigli V. Multicomponent flow modeling, modeling and simulation in science, engineering and technology. Birkhäuser: Boston; 1999.

[24] Poinsot T, Veynante D. Theoretical and numerical combustion, 2nd ed. R.T. Edwards; 2005. 